\title{
Definitive Assignment by Multigenome Analysis of the Gammaproteobacterial Genus Thermithiobacillus to the Class Acidithiobacillia
}

\author{
COREY M. HUDSON ${ }^{1}$, KELLY P. WILLIAMS ${ }^{1}$ and DONOVAN P. KELLY ${ }^{2, *}$
}

\author{
${ }^{1}$ Systems Biology Department, Sandia National Laboratories, Livermore, CA 94551, USA \\ ${ }^{2}$ School of Life Sciences, University of Warwick, Coventry CV4 7AL, UK
}

Submitted 13 March 2014, accepted 14 April 2014

Abstract

\begin{abstract}
The class Acidithiobacillia was established using multiprotein phylogenetic analysis of all the available genomes of the genus Acidithiobacillus (comprising Family I, the Acidithiobacillaceae, of the Acidithiobacillales, the order created for Bergey's Manual of Systematic Bacteriology), and for representative genomes of all available bacterial orders. The Acidithiobacillales contain a second family, the Thermithiobacillaceae, represented by Thermithiobacillus tepidarius, and created on the basis of nearest neighbour 16S ribosomal RNA gene sequence similarities. This could not be included in the original multiprotein analysis as no genome sequence for Thermithiobacillus was available. The publication of the genome sequence of Thermithiobacillus tepidarius in 2013 has enabled phylogenetic assessment of this organism by comparative multigenome analysis. This shows definitively that Thermithiobacillus is a member of the class Acidithiobacillia, distinct from the Acidithiobacillus genus, and confirms it to represent a second family within the Acidithiobacillia.
\end{abstract}

Ke y words: Thermithiobacillus, Acidithiobacillus, Acidithiobacillia, multigenome phylogeny; multiprotein analysis

Thermithiobacillus tepidarius is an obligately aerobic, moderately thermophilic, neutrophilic, obligately chemolithotrophic proteobacterium, initially classified as a strain of the betaproteobacterial genus Thiobacillus on the basis of its morphology and biochemistry (Wood and Kelly, 1985, 1986). It was subsequently redefined as the type species of the genus Thermithiobacillus (Kelly and Wood, 2000), and the type (and only) genus of the family Thermithiobacillaceae. This family was created using the proteobacterial phylogeny based on $16 \mathrm{~S}$ ribosomal RNA gene sequencing employed for the $2^{\text {nd }}$ edition of Bergey's Manual of Systematic Bacteriology (Garrity, Bell and Lilburn, 2005; Kelly and Wood, 2014a). It became Family II of the order Acidithiobacillales in the Gammaproteobacteria (Garrity, Bell and Lilburn, 2005). The earliest study of $16 \mathrm{~S}$ rRNA relationships among sulfur-oxidizing chemolithotrophs, using a partial sequence for Thermithiobacillus tepidarius, placed it midway between Thiobacillus neapolitanus and the extremely acidiphilic Thiobacillus thiooxidans in terms of evolutionary distances (Lane et al., 1992)). The adoption of $16 \mathrm{~S}$ rRNA gene sequence analysis as a major phylogenetic tool led to the reclassification of numerous species of Thiobacillus into a range of new genera, including Thermithiobacillus, or assignment to existing genera such as Thiomonas, Paracoccus and Acidiphilium (Kelly and Wood, 2000, 2005, 2014a, b; Kelly, Wood and Stackebrandt, 2005). While T.tepidarius was physiologically more like neutrophilic species of Thiobacillus, Halothiobacillus, and Thiomicrospira, or even the thermophile Thermothrix, its $16 \mathrm{~S}$ rRNA gene sequence shared only $85 \%$ identity with that of Thiobacillus thioparus, $82-85 \%$ with Halothiobacllus neapolitanus, H. halophilus, and H. kellyi, 79\% with Thiomicrospira crunogena, and 74-79\% with Thermothrix thiopara and $T x$. azorensis, unequivocally excluding it from those genera. The least distant phylogenetic relatives of Thermithiobacillus tepidarius are Acidithiobacillus species (comprising Family I, the Acidithiobacillaceae, of the Acidithiobacillales), with 90-91\% 16S rRNA gene sequence identity to At. thiooxidans and At.ferrooxidans. On the basis of nearest-neighbour 16S rRNA relationship, the parent order for Thermithiobacillus was the Acidithiobacillales, but it differed from these acidophiles in being thermophilic and neutrophilic, with only Acidithiobacillus caldus also being moderately thermophilic (Kelly and Wood, 2014b). It is not uncommon to find differences among 16s rRNA gene sequences of species of the same genus or family: for example, the range among Halothiobacillus species is $91-99 \%$ identity, but

\footnotetext{
* Corresponding author: D.P. Kelly, School of Life Sciences, University of Warwick, Coventry CV4 7AL, UK; e-mail: D.P.Kelly@ warwick.ac.uk
} 


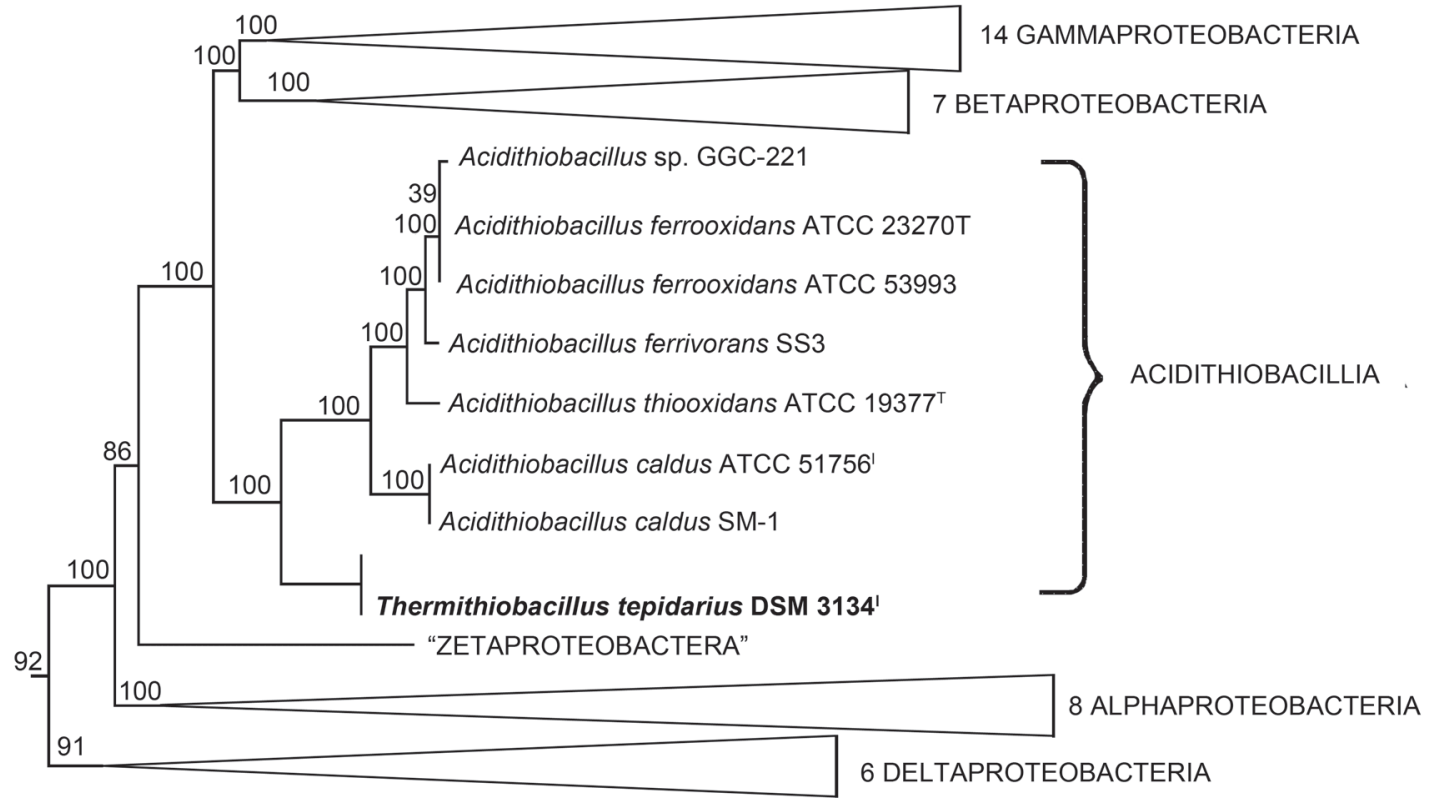

Fig. 1. Phylogenetic placement of Thermithiobacillus among the Acidithiobacillia. This portion of the full genome-scale maximumlikelihood analysis of 98 protein families, including each available member of the Acidithiobacillia, depicts several additional classes in collapsed form, using representative ("diagnostic") genera of Alpha-, Beta-, and Gammaproteobacteria, and roots this portion of the tree with the remaining bacteria and archaeal taxa, as shown in the full tree previously presented for the Acidithiobacillus species only (Williams and Kelly, 2013). Details of the 98 protein families and 135 genomes used in the analyses were listed previously (Williams and Kelly, 2013). Bootstrap support percentages are shown.

accompanied by significant physiological similarities. The 16S rRNA similarity of Thermithiobacillus to, but physiological divergence from Acidithiobacillus thus supported its retention as a separate family within the Acidithiobacillales. Clearly a more robust confirmation of this taxonomic relationship was desirable.

The Acidithiobacillales were classed as Gammaproteobacteria until genome-scale phylogenetic analyses of Acidithiobacillus showed that this genus could not be assigned to any previously defined class (Williams et al., 2010; Williams and Kelly, 2013). Our expanded multigenome study, using all the available genomes of Acidithiobacillus species, led to the creation of a seventh proteobacterial class, the Acidithiobacillia (Williams and Kelly, 2013). The initial study trialled 1891 prokaryotic genomes, including 104 gammaproteobacterial genomes: 356 protein families were analyzed and compared with those encoded by alpha- and betaproteobacterial genomes, resulting in the exclusion of Acidithiobacillus from the Gammaproteobacteria (Williams et al., 2010). A study of the ubiquitous ribosomal proteins of 995 bacteria by Yutin et al. (2012) also placed At. ferrooxidans outside 251 species of Gamma-, 75 species of Beta-, and 180 species of Alpha-, Delta-, and Epsilonproteobacteria, and to be located in the root of the Betaand Gammaproteobacteria. When the class Acidithiobacillia was created, no genome sequence was available for Thermithiobacillus, so its membership of the class could not be definitively tested. The genome sequence of
Thermithiobacillus tepidarius DSM 3134 became available from the Joint Genome Institute in 2013 (http:// img.jgi.doe.gov/cgi-bin/w/main.cgi?section=Taxon Detail\&page=taxonDetail\&taxon_oid=2523533554), and shown to contain $2.96 \mathrm{Mb}$, which is within the range of $2.83-3.02 \mathrm{Mb}$ for Acidithiobacillus species (Kelly and Wood, 2014b). This has now enabled us to include Thermithiobacillus in the multigenome dataset that was used to create the Acidithiobacillia class.

The protocol for the present multigenome study was as described previously (Williams et al., 2010; Williams and Kelly, 2013), and began with the "phase 2" dataset used in the demonstration of the Acidithiobacillia (Williams and Kelly, 2013). This dataset comprised 11,949 protein sequences from 98 broadly-distributed protein families, from 135 taxa, including six Archaea, seven Acidithiobacillus strains, and 122 diverse additional bacteria (Williams and Kelly, 2013). For each of the 98 protein families, a Thermithiobacillus tepidarius member was identified using the hidden Markov model approach implemented in HMMER v. 3.0 (Finn, Clements and Eddy, 2011). The protein family sequences were freshly aligned, masking ambiguously-aligned regions, and concatenated into a partitioned amino-acid sequence supermatrix that was subjected to a maximum-likelihood tree search with 1000 bootstraps as described previously (Williams et al., 2010; Williams and Kelly, 2013). This analysis (Fig. 1) unambiguously placed Thermithiobacillus tepidarius into the Acidithiobacillia, distinct from 
the Acidithiobacillus strains, and recapitulated the previous placement of the class, as a sister to the combined Gammaproteobacteria and Betaproteobacteria, and subtended by the 'Zetaproteobacteria' (Williams and Kelly, 2013). We conclude that Thermithiobacillus is a valid member of the class Acidithiobacillia, and should be retained in the class as the type genus of the family Thermithiobacillaceae. From the data currently available, we do not propose further division of the class, although the Acidithiobacillus and Thermithiobacillus species might in due course justify separation at the order level. Moreover, the confirmation of the taxonomic location of Thermithiobacillus by this multigenome analysis illustrates both the reliability of 16S rRNA phylogeny as a diagnostic tool, and the power of the multigenome analysis procedure at the genus, family and class levels. The multigenome method could become a useful new tool, especially for discrimination among families, orders, and classes of prokaryotes. This would require the eventual establishment of a public database of multiprotein sequences and computational tools.

Currently, there is only one established species of Thermithiobacillus (DSM 3134), but strain NCIMB 8349 (isolated as Thiobacillus thioparus strain ParkerM) is also a strain of Thermithiobacillus (Boden et al., 2012; Kelly and Wood, 2014a), showing 99.5\% 16S rRNA gene sequence identity to that of the type strain (accession numbers HM173631 and AJ459801): the genome of this strain is currently being sequenced (R. Boden, personal communication). Three other strains have been reported from Korea (a moderate thermophile from mine wastewater; Chang et al., 1997) and China (from a sewage treatment plant; K. Gao, H.L. Yang and S.S. Feng, unpublished data). The 16S rRNA gene sequences of these strains (AF02364, KC478673, KC493561) show $98.3-99.4 \%$ identity to that of the type strain and $99-100 \%$ identity to that of NCIMB 8349 , suggesting that the family represented by this genus is widely distributed and may contain several species.

\section{Acknowledgements}

Work at Sandia was supported by a Laboratory Directed Research and Development award. Sandia National Laboratories is a multi-program laboratory managed and operated by Sandia Corporation, a wholly-owned subsidiary of Lockheed Martin Corporation, for the U.S. Department of Energy's National Nuclear Security Administration under contract DE-AC04-94AL85000.

We thank Ann Wood (King's College London), Rich Boden (Plymouth University), and Nikos Kyrpides (DOE Joint Genome Institute) for advice about Thermithiobacillus.

\section{Literature}

Boden R., D. Cleland, P.N. Green, Y. Katayama, Y. Uchino, J.C. Murrell and D.P. Kelly. 2012. Phylogenetic assessment of culture collection strains of Thiobacillus thioparus, and definitive $16 \mathrm{~S}$ rRNA gene sequences for T. thioparus, T. denitrificans, and Halothiobacillus neapolitanus. Arch. Microbiol. 194: 187-195.

Chang S.Y., J.S. Yoon, Y.K. Shin, Y-H. Park, J.Y. Park, S.S. Yang, M-J. Koh, S.M. Yoon, J.S. Lee, I.H. Lee and S.Y. Kim. 1997. Isolation, characterization, and phylogenetic position of a new sulfuroxidizing bacterium. J. Microbiol. (Korea) 35: 165-171.

Finn R.D., J. Clements and S.R. Eddy. 2011. HMMER web server: interactive sequence similarity searching. Nucleic Acids Res. 39: W29-37.

Garrity G.M., J.A. Bell and T. Lilburn. 2005. Order II. Acidithiobacillales ord. nov. p. 60. In: Bergey's Manual of Systematic Bacteriology, vol. 2B, $2^{\text {nd }}$ edition. Brenner, D.J., N.R. Krieg, J.T. Staley and G.M. Garrity (eds). New York, Springer.

Kelly D.P. and A.P. Wood. 2000. Reclassification of some species of Thiobacillus to the newly designated genera Acidithiobacillus gen. nov., Halothiobacillus gen. nov. and Thermithiobacillus gen. nov. Int. J. Syst. Evol. Microbiol. 50: 511-516.

Kelly D.P. and A.P. Wood (2005) Genus I. Thermithiobacillus Kelly and Wood. pp. 62-63. In: Bergey's Manual of Systematic Bacteriology, vol. 2B, $2^{\text {nd }}$ edition. Brenner D.J., N.R. Krieg, J.T. Staley and G.M. Garrity (eds). New York, Springer.

Kelly D.P. and A.P. Wood (2014a) The family Thermithiobacillaceae. In: The Prokaryotes, vol. 9, $4^{\text {th }}$ edition. Stackebrandt E., E. Rosenberg, E. Delong, S. Lory and F. Thompson (eds). New York and Heidelberg, Springer (in press).

Kelly D.P. and A.P. Wood (2014b) The family Acidithiobacillaceae. In: The Prokaryotes, vol. 9, $4^{\text {th }}$ edition. Stackebrandt, E., E. Rosenberg, E. Delong, S. Lory and F. Thompson (eds). New York and Heidelberg, Springer (in press).

Kelly D.P., A.P. Wood and E. Stackebrandt. 2005. Genus II. Thiobacillus Beijerinck 1904. pp. 764-769. In: Bergey's Manual of Systematic Bacteriology, vol. 2C, $2^{\text {nd }}$ edition. Brenner, D.J., N.R. Krieg, J.T. Staley and G.M. Garrity (eds). New York, Springer.

Lane D.J., A.P. Harrison, D. Stahl, B. Pace, S.J. Giovannoni, G.J. Olsen and N.R. Pace. 1992. Evolutionary relationships among sulfur- and iron-oxidizing eubacteria. J. Bacteriol. 174: 269-278.

Williams K.P. and D.P. Kelly. 2013. Proposal for a new class within the phylum Proteobacteria, Acidithiobacillia classis nov., with the type order Acidithiobacillales, and emended description of the class Gammaproteobacteria. Int. J. Syst. Evol. Microbiol. 63: 2901-2906.

Williams K.P., J.J. Gillespie, B.W. Sobral, E.K. Nordberg, E.E. Snyder, J.M. Shallom and A.W. Dickerman. 2010. Phylogeny of Gammaproteobacteria. J. Bacteriol. 192: 2305-2314.

Wood A.P. and D.P. Kelly. 1985. Physiological characteristics of a new thermophilic obligately chemolithotrophic Thiobacillus species, Thiobacillus tepidarius. Int. J. Syst. Bacteriol. 35: 434-437.

Wood A.P. and D.P. Kelly. 1986. Chemolithotrophic metabolism of the newly-isolated moderately thermophilic, obligately autotrophic Thiobacillus tepidarius. Arch. Microbiol. 144: 71-77.

Yutin N., P. Puigbò, E.V. Koonin and Y.I. Wolf. 2012. Phylogenomics of prokaryotic ribosomal proteins. PLoS ONE 7(5) e36972. (doi:10.1371/journal.pone.0036972). 
